\title{
Does the number of implants have any relation with peri-implant disease?
}

\author{
Bernardo Born PASSONI', Haline Renata DALAGO'1, Guenther SCHULDT FILHO'1, João Gustavo OLIVEIRA DE \\ SOUZA ${ }^{1}$, César Augusto Magalhães BENFATTI ${ }^{2}$, Ricardo de Souza MAGINI ${ }^{3}$, Marco Aurélio BIANCHINI ${ }^{3}$
}

\footnotetext{
1- Department of Implant Dentistry, Federal University of Santa Catarina, Florianópolis, SC, Brazil.

2- Department of Surgery, Federal University of Santa Catarina, Florianópolis, SC, Brazil.

3- Department of Periodontics, Federal University of Santa Catarina, SC, Brazil.
}

Corresponding address: Bernardo Born Passoni - R. Esteves Junior, 463/701 - Centro - Florianópolis - SC - Brazil - $88015-130$ - Phone: +55 48 37219077 - e-mail: bpassoni@hotmail.com

Submitted: February 6, 2014 - Modification: June 15, 2014 - Accepted: June 18, 2014

\section{ABSTRACT}

\begin{abstract}
$\mathrm{O}$ bjective: The aim of this study was to evaluate the relationship between the number of pillar implants of implant-supported fixed prostheses and the prevalence of periimplant disease. Material and Methods: Clinical and radiographic data were obtained for the evaluation. The sample consisted of 32 patients with implant-supported fixed prostheses in function for at least one year. A total of 161 implants were evaluated. Two groups were formed according to the number of implants: G1) $\leq 5$ implants and G2) >5 implants. Data collection included modified plaque index (MPi), bleeding on probing (BOP), probing depth (PD), width of keratinized mucosa (KM) and radiographic bone loss (BL). Clinical and radiographic data were grouped for each implant in order to conduct the diagnosis of mucositis or peri-implantitis. Results: Clinical parameters were compared between groups using Student's t test for numeric variables (KM, PD and BL) and Mann-Whitney test for categorical variables (MPi and BOP). KM and BL showed statistically significant differences between both groups ( $p<0.001)$. Implants from G1 - $19(20.43 \%)$ - compared with G2 - $26(38.24 \%)$ - showed statistically significant differences regarding the prevalence of peri-implantitis $(p=0.0210)$. Conclusion: It seems that more than 5 implants in total fixed rehabilitations increase bone loss and consequently the prevalence of implants with periimplantitis. Notwithstanding, the number of implants does not have any influence on the prevalence of mucositis.
\end{abstract}

Keywords: Peri-implantitis. Mucositis. Dental implants.

\section{INTRODUCTION}

Routine treatment of edentulism with fixed prostheses supported by osseointegrated fixtures appears to be a highly efficient method, giving predictable long-term results in edentulous patient populations ${ }^{1}$.

Peri-implant diseases are one of factors responsible for implant failures. These lesions are commonly asymptomatic and frequently detected in follow-up visits. The presence of increased probing depth $(\geq 5 \mathrm{~mm})^{20,28}$ bleeding on probing and/or pus is a key factor that may facilitate the diagnosis of peri-implantitis. Yet, peri-implantitis is characterized mainly by the presence of progressive bone loss which occurs after the biological response associated with the adaptation phase adjacent to the implant ${ }^{2,22}$. The term mucositis is related to the inflammatory lesion induced by nonspecific biofilm ${ }^{18}$ limited to peri-implant mucosa without involvement of bone tissue ${ }^{13}$.

Based on clinical findings, radiographs can be useful for the confirmation of the presence or absence of the disease ${ }^{19}$. In the absence of previous radiographic records, a threshold vertical distance of $2 \mathrm{~mm}$ from the expected marginal bone level following remodelling post-implant placement is recommended, provided peri-implant inflammation is evident ${ }^{28}$. Thus, both the bone remodeling that occurs after exposure of the implant to the oral environment (saucerization) and the late bone loss characterized by gradual loss of marginal bone after 
osseointegration has been consolidated ${ }^{31}$ must be included when evaluating the success of implants ${ }^{6}$. As seen in a particular study, gradual bone loss of $0.2 \mathrm{~mm}$ after the first year of function can be considered successful. For this reason, a bone loss $<2.4 \mathrm{~mm}$ would be acceptable during the first five years of function ${ }^{3}$.

Moreover, peri-implant diseases (mucositis or peri-implantitis) are responsible for several implant losses and, if not treated, may lead to failure of bone healing around the titanium surface ${ }^{11}$.

Peri-implant diseases might be considered an imbalance of the action of specific Gram-negative bacteria and spirochetes ${ }^{33}$ against host organism, caused by a decrease in immunity. They can affect only the mucosa (mucositis) and also the supporting bone, which characterizes peri-implantitis ${ }^{18}$. These can be developed after the implants are exposed to the oral environment and masticatory loads for a period (at least 1 year in our research) and should be considered especially after the formation phase of the biological distances. Our study considered that implants with peri-implantitis had to present probing depth $\geq 5 \mathrm{~mm}$, at least one point with bleeding/suppuration on probing and radiographic bone loss $>2 \mathrm{~mm}$.

Based on clinical longitudinal studies, the time of prosthesis installation should be chosen to establish baseline criteria. To establish a baseline, a radiograph should be obtained to determine alveolar bone levels after physiologic remodeling, and peri-implant probing assessments should be performed ${ }^{17}$.

Regarding patients' hygienic procedures, in order to maintain the implant healthy, it's crucial to stimulate and orientate them. These procedures should be performed by the patient under professional supervision ${ }^{19}$. Still, the understanding between the dentist and the technician may facilitate prostheses production with less plaque accumulation potential ${ }^{30}$. In this context, it is suggested that the lowest amount of implants in total rehabilitation can favor the homeostasis of peri-implant tissues, mainly by the distribution/ position of the implants and their relationship with the prosthetic piece.

What remains unclear in the literature is whether the number of implants has any influence on the health status of the implants. Thus, the aim of this study was to evaluate the relationship between the number of pillar implants ( $\leq 5$ and $>5$ ) of implant-supported fixed prostheses and the prevalence of peri-implant diseases (mucositis and peri-implantitis).

\section{MATERIAL AND METHODS}

\section{Sample selection}

This study was approved by the ethics committee for clinical studies of Federal University of Santa Catarina, Florianópolis-SC, Brazil (Protocol no. 128/2006). Each patient read and signed an informed consent form before entering the study. The patients included in the study were those who had received osseointegrated implants with an external hex $4.1 \mathrm{~mm}$ connection and fixed metal with resin prostheses supported by implants done at the Center of Teaching and Research in Dental Implants, placed in the Center of Sciences and Health (CCS) of the Federal University of Santa Catarina (UFSC) from 2004 to 2010. Participants were selected from a total of 35 patients that had implant-supported fixed prostheses and received the implants from 2004 to 2010. The individuals excluded from the study were the ones with radiographs unable to be measured, patients with psychiatric disorders and those who participated in any follow-up control for plaque removal. Thus, 32 patients were selected - 14 men and 18 women who had 161 implants in function for at least one year. However, at first the implants were evaluated in groups according to quantity only, without considering its location.

\section{Data collection}

Patient examination and collection of all data were blind, performed by an independent and experienced clinician. All prostheses on implants were resin complete dentures with metal substructures and were removed in order to facilitate data collection. The cantilever evaluation was completed by considering the length of the cantilever. For this evaluation, the study was divided into two groups: G1) $\leq 5$ implants and G2) $>5$ implants as implant-supported fixed prostheses pillars. Moreover, the following data were recorded for each implant after the prosthesis' removal.

- Modified plaque index (MPi) ${ }^{24}$ - (0: no plaque, 1: detected with the point of the instrument, 2: visual plaque, 3 : excessive plaque accumulation).

- Modified bleeding on probing index (BOP) ${ }^{24}$ ( 0 : no bleeding, 1: bleeding spots, 2: thin line of blood around the implant, 3: excessive bleeding).

- Hygiene difficulty - ranked by the patient as high, medium or low.

- Probing depth (PD) 20,28.

- Marginal recession - (0: absent, 1: $\leq 1 \mathrm{~mm}$, $>2 \mathrm{~mm}$ ).

- Width of keratinized mucosa (KM) - (Differences in color, texture and mobility served as markers for mucogingival junction detection $)^{21}$.

- Distance between implants - classified as $\geq 3$ $\mathrm{mm}$ or $<3 \mathrm{~mm}$. 
- Radiographic bone loss (BL) ${ }^{30,34}$ - measured by comparing the periapical radiographs. Bone level was measured from the implant platform to the first bone-implant contact (in $\mathrm{mm}$ ).

- Dental arch positioning (in $\mathrm{mm}$ ) - anterior (incisors and canines) or posterior (pre molars and molars).

- Maxillary positioning - superior (maxilla) or inferior (mandible).

- Cantilever distal extension - (1: >10 mm and 2: $\leq 10 \mathrm{~mm})$.

\section{Radiographic analysis}

The distance between implant platform and the first bone-implant visible contact was measured in millimeters at the mesial and distal aspect of each implant using periapical radiographs. Special care was taken to position the film parallel to the fixture to provide an optimal and undistorted image ${ }^{9}$. The images were digitalized and the bone loss was evaluated in the Digimizer image analysis software ${ }^{\circledR}$ (MedCalc Software, Ostend, Belgium). For each implant, the highest value for bone loss found at the mesial or distal aspect was used. Further care was taken to ensure that threads on both the mesial and distal sides of the implants were clearly visible ${ }^{12}$. All the images were analyzed by the same examiner.

\section{Statistical analysis}

Data were digitalized and organized for comparison between groups and analyses of the results. Clinical parameters were compared between groups. Student's t test was applied for numeric variables ( $K M, P D, B L)$. For $K M$ and $P D$, a mean value (in millimeters) related to all four examined areas was calculated. For $\mathrm{BL}$, the analysis considered the greatest value in millimeters. Categorical variables such as DP and BOP were evaluated using Mann-Whitney test. Chi-square test was used for comparison and statistical analysis for binary variables - prevalence of mucositis and peri-implantitis. Furthermore, Yates correction was applied to adjust the value of the chi-square test when the expected frequency by the law of averages was less than 5. For insufficient samples to perform the chi-square or Yates correction, Fisher's test was applied. P value $\leq 0.05$ was considered to indicate a statistically significant difference between analyzes.

\section{Diagnosis}

The diagnosis of the implants was performed at the time of the follow-up visit. In order to be considered with $\mathrm{PI}$, implants had to present PD $\geq 5$ $\mathrm{mm}$, at least one point of bleeding/suppuration on probing (BOP) and $\mathrm{BL}>2 \mathrm{~mm}$. For $\mathrm{PD}$, the highest value was considered. Parameters for $P D$ and $B O P$ were obtained using a periodontal probe (PCV12PT Hu-Friedy Inc., Chicago, IL, USA). Measurements were performed by a single calibrated professional in order to reduce errors and establish reliability and consistency. Also, all prostheses were removed prior to the examination to permit data collection.

\section{RESULTS}

Implants were in function for at least one year. The mean time in function was 47.46 months for $\mathrm{G} 1$ and 67.56 months for $\mathrm{G} 2$. From the total implant fixed prosthesis, $14(43.75 \%)$ were located in the maxilla and $18(56.25 \%)$ in the mandible. Regarding the number of implants, 75 (46.58\%) and $86(53.42 \%)$ were located in the maxilla and mandible, respectively (Table 1 ). Patients' age ranged from 45 to 80 years (mean age 63.43). For time in function, implants had an average of 55.95 months with a standard deviation of 24.21 (12.48 to 64.79 months). The average of implants per patients was 5.96.

Evaluating the amount of KM, G1 showed lower height compared to $G 2(p<0.001)$. The results were similar in assessing DP $(p=0.31), P D(p=0.99)$ and BOP $(p=0.46)$ between groups, showing no

Table 1- Frequency and distribution of data on the G1 implants (up to 5 implants) and G2 (more than 5 implants) according the implant placement area

\begin{tabular}{ccccc}
\hline G1 & Implants $\mathbf{n}(\%)$ & Healthy & Mucositis & Peri-implantitis \\
\hline Post-sup & $10(6.21 \%)$ & $1(6.25 \%)$ & $7(7.00 \%)$ & $2(4.44 \%)$ \\
\hline Ant-sup & $16(9.94 \%)$ & $1(6.25 \%)$ & $11(11.00 \%)$ & $4(8.89 \%)$ \\
\hline Post-inf & $25(15.53 \%)$ & $3(18.75 \%)$ & $17(17.00 \%)$ & $5(11.11 \%)$ \\
\hline Ant-inf & $42(26.09 \%)$ & $4(25.00 \%)$ & $25(25.00 \%)$ & $13(28.89 \%)$ \\
\hline G2 & & & \\
Post-sup & $25(15.53 \%)$ & $2(12.50 \%)$ & $17(17.00 \%)$ & $6(13.33 \%)$ \\
\hline Ant-sup & $24(14.91 \%)$ & $2(12.50 \%)$ & $13(13.00 \%)$ & $9(20.00 \%)$ \\
Post-inf & $6(3.73 \%)$ & $1(6.25 \%)$ & $4(4.00 \%)$ & $1(2.22 \%)$ \\
\hline Ant-inf & $13(8.07 \%)$ & $2(12.50 \%)$ & $6(6.00 \%)$ & $5(11.11 \%)$ \\
\hline Total & $161(100.00 \%)$ & $16(100.00 \%)$ & $100(100.00 \%)$ & $45(100.00 \%)$ \\
\hline
\end{tabular}


Table 2- Frequency and distribution of data in G1 (up to 5 implants) and G2 (more than 5 implants) according keratinized mucosa $(\mathrm{KM})$, plaque (DP), probing depth (PD), bleeding probing (BOP) and bone loss (BL). SD: standard deviation. * Statistically significant

\begin{tabular}{|c|c|c|c|c|c|}
\hline \multirow[b]{2}{*}{$\begin{array}{c}\text { Clinical } \\
\text { Parameters }\end{array}$} & \multicolumn{2}{|c|}{ G1 } & \multicolumn{2}{|c|}{ G2 } & \multirow[b]{2}{*}{$P$ value } \\
\hline & Mean & DP & Mean & DP & \\
\hline $\mathrm{KM}$ & 2.53 & 1.40 & 3.66 & 1.80 & $<0.001^{*}$ \\
\hline DP & 1.76 & 1.11 & 1.62 & 1.05 & 0.3112 \\
\hline $\mathrm{PD}(\mathrm{mm})$ & 2.81 & 0.92 & 2.81 & 0.93 & 0.9929 \\
\hline BOP & 1.89 & 1.25 & 1.74 & 1.15 & 0.4600 \\
\hline $\mathrm{BL}(\mathrm{mm})$ & 2.71 & 1.10 & 3.76 & 1.43 & $<0.001^{*}$ \\
\hline
\end{tabular}

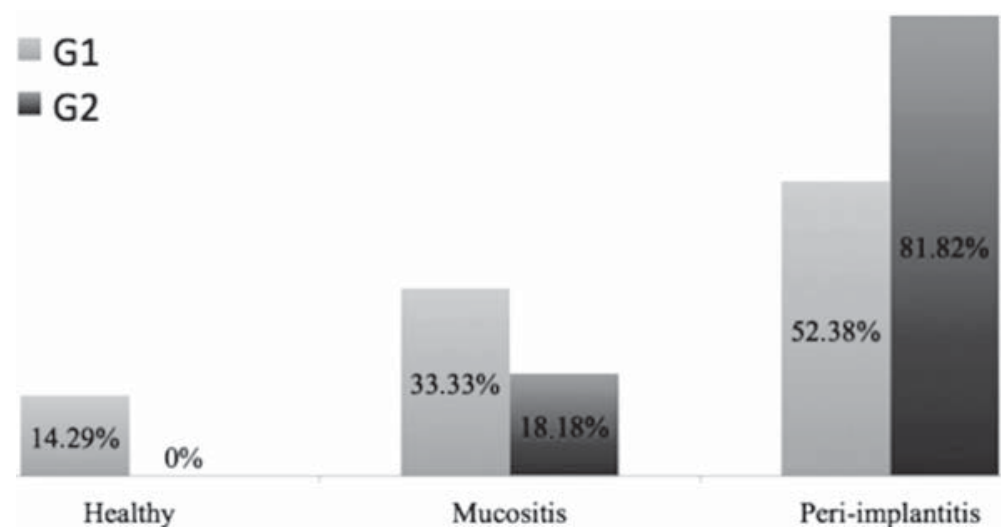

Figure 1- Distribution of healthy, mucositis and peri-implantitis patients between $\mathrm{G} 1$ (fixed denture supported by up to 5 implants) and G2 (fixed denture supported by more than 5 implants)

statistically significant difference. In contrast, the results of $B L$ were higher for $G 2(p<0.001)$. The frequency and distribution of data from implants in $\mathrm{G} 1$ and $\mathrm{G} 2$ for $\mathrm{MPi}, \mathrm{BOP}, \mathrm{PD}, \mathrm{KM}$ and $\mathrm{BL}$ are displayed in Table 2.

Regarding the prevalence of peri-implant diseases, the study showed $3(9.38 \%)$ healthy patients, $9(28.13 \%)$ patients with mucositis and $20(62.50 \%)$ with peri-implantitis. There was no statistical difference between groups, and all healthy patients were in $\mathrm{G} 1(\mathrm{p}=0.49)$. There were 7 patients diagnosed with mucositis (33.33\%) in $\mathrm{G} 1$ and $2(18.18 \%)$ patients in $\mathrm{G} 2(\mathrm{p}=0.62)$. For peri-implantitis, $11(52.38 \%)$ patients in $\mathrm{G} 1$ and 9 $(81.82 \%)$ patients in $\mathrm{G} 2(\mathrm{p}=0.21)$. The distribution of healthy patients, mucositis and peri-implantitis between $\mathrm{G} 1$ and $\mathrm{G} 2$ can be seen in Figure 1.

From all implants evaluated, $16(9.93 \%)$ were healthy, $11(11.82 \%)$ in $\mathrm{G} 1$ and $5(7.35 \%)$ in $\mathrm{G} 2$ $(p=0.5)$. Regarding the prevalence of mucositis between the implants present in each group, there was no statistical difference between G1 and G2, which presented 63 implants (67.74\%) and 37 implants $(54.41 \%)$ with this condition $(p=0.11)$. As for peri-implantitis, however, G1 showed lower prevalence of 19 implants (20.43\%) while G2 had 26 implants $(38.24 \%)$ with this condition $(p=0.02)$.
When the regions of implant placement were compared separately, only the anterior-inferior region had higher rates of peri-implantitis in $\mathrm{G} 1$ compared to G2; however, it was not statistically significant (Table 1 ).

\section{DISCUSSION}

The survival of implant-supported fixed prostheses by a smaller number of implants shows good results both in the mandible and in the maxilla ${ }^{23}$. In this context, it is suggested that the fewer implants in total rehabilitation, the better. Yet, a smaller number of implants may enhance the homeostasis of peri-implant tissues, mainly by the distribution/position of the implants and their relationship with the prosthetic piece.

Peri-implant health can be maintained over the long term even in areas with absence of $\mathrm{KM}$, provided that suitable plaque control is performed ${ }^{5,15}$. Other authors disagree and suggest that $\mathrm{KM}$ is related to a reduced accumulation of plaque and mucosal inflammation 7 . In our study, although $\mathrm{G} 2$ presents a statistically greater range of KM compared to G1, a larger band of KM was not enough to prevent periimplantitis, since $\mathrm{G} 2$ showed higher rates of $\mathrm{BL}$. Interestingly, the lack of KM seems to negatively 
influence mucositis. It is explained by the fact that G1 showed higher rates this disease. This result corroborates with another study that reports that areas with higher amount of keratinized mucosa show higher incidence of mucositis ${ }^{27}$. This may be related to hygiene difficulty in the absence of $\mathrm{KM}^{10}$, but the accumulation of bacteria is not always the cause of peri-implantitis, a factor that might explain why a higher rate of mucositis did not provide a higher rate of peri-implantitis in G1. However, there is no parameter able to predict whether this range is necessary or not ${ }^{14}$.

Greater BL was observed in the implants of $\mathrm{G} 2$ patients in comparison to $\mathrm{G} 1$. This might have been a result of the difficulties of hygiene because of the larger area for plaque control. In a recent epidemiological study, it was stated that partial or full dentures are respectively 1.83 and 2.44 times more likely to have bone loss bigger than $2 \mathrm{~mm}$ that unitary prostheses. This may be due to the difficulty of hygiene in the junction of the implants from partial or full dentures ${ }^{31}$. Moreover, another factor taken into consideration is the improper positioning of the implants. Usually, when placed very close to each other, it might hinder hygiene procedures and compromise peri-implant biological distances ${ }^{32}$.

Group 2 showed $100 \%$ of patients with mucositis. As for implants analyzed separately, results showed prevalence of peri-implantitis similar to the one found in the literature $(12 \% \text { to } 40 \%)^{16}-\mathrm{G} 1$ and G2 with $20.43 \%$ to $38.24 \%$. Regarding patients, $50 \%$ had peri-implantitis in the $\mathrm{G} 1$ and $81.8 \%$ in the $\mathrm{G} 2$. The $\mathrm{G} 2$ values were higher than previous reports $(28 \% \text { to } 56 \%)^{16}$. Differences in prevalence between patients and implants can be explained by the fact that only one implant disease can is enough to categorize patients as sick or healthy.

Measurement of bone level throughout time is a valuable indicator for evaluating clinical performance of dental implants. This is because the gradual and undiagnosed bone loss leads to loss of the implant. Radiographic monitoring of bone changes should be analyzed with caution, since the pattern of bone loss varies among individuals ${ }^{13}$. Thus, both bone remodeling - after exposure of the implant to the oral environment - and late bone loss should be included in evaluating the success of implants ${ }^{4}$. Bone loss in the first year in function was considered acceptable if up to $2 \mathrm{~mm}^{9}$; therefore, this study established diagnosis of peri-implantitis if probing depth was higher or equal to $5 \mathrm{~mm}$, associated with radiographic bone loss higher than $2 \mathrm{~mm}$. This bone loss can be related to bacterial and iatrogenic factors such as bad positioning of implant placement and noncompliance with the minimum distances between them to form biological distances. According to clinical observations of large plaque accumulation, these data lead to the empirical belief that the higher the amount of implants, the bigger the hygiene difficulty and, consequently, the higher the bone loss. Also, smoking habits in combination with poor compliance and poor oral hygiene appears to enhance the risk of peri-implantitis ${ }^{26}$.

Although the results have been presented above, in the analysis of some peri-implant disease, it should be considered that the patients in this study had only total rehabilitations. Indeed, this might have influenced the appearance of the lesions, since the prosthetic piece can limit or hinder the control of plaque, mainly in the individuals with the higher number of implants.

Even if some studies indicate that more frequent follow-up visits are better for prevention, more studies are necessary to confirm that statement. For this study, it was opted not to undertake patients to a follow-up program because it is not the reality for all patients using implant-supported fixed prostheses ${ }^{29}$.

Low motivation and difficulty of cleaning can be considered main factors in the development of peri-implant disease, since they may cause the larger dental plaque accumulation. This reduced motivation is justified by motor difficulty of the patient and the design of the inner portion of the prosthesis. The presence of concave shapes in the inner portion of the prosthesis and intimate contact with the mucosa provide a larger plaque accumulation and hinder the use of interdental brush, because it traumatizes the mucosa of the patient. Nevertheless, it must be considered that an peri-implant injury may have started and/or be active by iatrogenic factors such as excess cement, inappropriate emerging profile of the prosthesis, inadequate pillars, incorrectly positioned implants and complications in laboratory stages ${ }^{20}$. And this might also have been the result of overloading and/or difficulty of hygiene due to a larger area for bacterial plaque control, or misplaced implants that may hamper hygiene procedures ${ }^{31}$.

Renvert, et al. ${ }^{25}$ (2011) state that the risk of periimplantitis increases with age, and this is probably related to the motor difficulty of the patients. However, on the other hand, a novel study showed that in those aged $>60$ years old $(18.63 \%)$, the prevalence is lower if compared with those being $\leq 60$ years $(44.07 \%)^{25}$.

Thus, the motivation of the patient to perform correct hygiene ${ }^{8}$, reduced number of implants, the correct positioning of implants and integrated planning between dentist and prosthetic favor the manufacturing of a suitable prosthesis and especially peri-implant health.

In conclusion, within the limitations of this study, total rehabilitations supported by up to 
five implants seem to have lower bone loss and are associated with lower prevalence of implants with peri-implantitis. However, it is observed that the number of implants does not influence the prevalence of mucositis.

\section{REFERENCES}

1- Adell R, Eriksson B, Lekholm U, Brånemark P-I, Jemt T. A long-term follow-up study of osseointegrated implants in the treatment of totally edentulous jaws. Int J Oral Maxillofac Implants. 1990;5:347-59.

2- Albrektsson T, Buser D, Chen ST, Cochran D, DeBruyn H, Jemt $\mathrm{T}$, et al. Statements from the Estepona consensus meeting on peri-implantitis. Clin Implant Dent Relat Res. 2012;14:781-2.

3- Albrektsson T, Isidor F. Consensus report of session IV. In: Lang NP, Karring, T, eds. Proceedings of the First European Workshop on Periodontology. Chicago: Quintessence; 1994. p. 365-9.

4- Albrektsson T, Zarb G, Worthington P, Eriksson AR. The longterm efficacy of currently used dental implants: a review and criteria of success. Int J Oral Maxillofac Implants. 1986;1:11-25. 5- Bengazi, F, Wennström JL, Lekholm U. Recession of the soft tissue margin at oral implants. A 2-year longitudinal prospective study. Clin Oral Implants Res. 1996;7:303-10.

6- Berglundh T, Lindhe J, Ericsson I, Marinello CP, Liljenberg $B$, Thomsen P. The soft tissue barrier at implants and teeth. Clin Oral Implant Res. 1991;2:81-90.

7- Boynueğri D, Nemli SK, Kasko IA. Significance of keratinzed mucosa around dental implants: a prospective comparative study. Clin Oral Implants Res. 2013;24(8):928-33.

8- Corbella S, Dell Fabbro M, Taschieri S, De Siena F, Francetti L. Clinical evaluation of an implant maintenance protocol for the prevention of peri-implant diseases in patients treated with immediately loaded full-arch rehabilitations. Int J Dent Hyg. 2011;9:216-22.

9- De Bruyn $H$, Van de Velde T, Collaert B. Immediate functional loading of TiOblast dental implants in full-arch edentulous mandibles: a 3-year prospective study. Clin Oral Implants Res. 2008; 19:717-23.

10- Esposito M, Hirsch JM, Lekholm U, Thomsen P. Biological factors contributing to failures of osseointegrated oral implants.

(I) Success criteria and epidemiology. Eur J Oral Science. 1998;106:527-55.

11- Esposito M, Hirsch JM, Lekholm U, Thomsen P. Biological factors contributing to failures of osseointegrated oral implants (II). Etiopathogenesis. Eur J Oral Sci. 1998;106:721-64.

12- Fischer K, Stenberg T, Hedin M, Sennerby L. Five-year results from a randomized, controlled trial on early and delayed loading of implants supporting full-arch prosthesis in the edentulous maxilla. Clin Oral Implants Res. 2008;19:433-41.

13- Fransson C, Tomasi C, Pikner S, Gröndahl K, Wennström $\mathrm{JL}$, Leyland $\mathrm{AH}$, et al. Severity and pattern of peri-implantitisassociated bone loss. J Clin Periodontol. 2010;37:442-8.

14- Greenstein G, Cavallaro J. The clinical significance of keratinized gengiva around dental implants. Comp Cont Educ Dent. 2011;32:24-34.

15- Heckmann SM, Schrott A, Graef F, Wichmann MG, Weber HP. Mandibular two-implant telescopic overdentures. Clin Oral Implants Res. 2004;15:560-9.

16- Heitz-Mayfield LJ. Peri-implant diseases: diagnosis and risk indicators. J Clin Periodontol. 2008;35:292-304.
17- Heitz-Mayfield LJ. Prevention and non-surgical therapy of peri-implant disease: Surgical therapy of peri-implantitis. Forum Implantologicum. 2008;4:74-8.

18- Heitz-Mayfield LJ, Lang NP. Comparative biology of chronic and aggressive periodontitis vs. peri-implantitis. Periodontol 2000. 2010;53:167-81.

19- Klinge B, Gustafsson A, Berglundh T. A systematic review of the effect of anti-infective therapy in the treatment of peri-implantitis. J Clin Periodontol. 2002;29:213-25.

20- Lang NP, Berglundh T, Working Group 4 of Seventh European Workshop on Periodontology. Periimplant diseases: where are we now? Consensus of the Seventh European Workshop on Periodontology. J Clin Periodontol. 2011;38:178-81.

21- Lin GH, Chan HL, Wang HL. The significance of keratinized mucosa on implant health: a systematic review. J Periodontol. 2013;84:1755-67.

22- Lindhe J, Meyle J, Group D of European Workshop on Periodontology. Peri-implant diseases: Consensus Report of the Sixth European Workshop on Periodontology. J Clin Periodontol. 2008;35:282-5.

23- Maló P, Nobre MD, Lopes A. The rehabilitation of completely edentulous maxillae with different degrees of resorption with four or more immediately loaded implants: a 5-year retrospective study and a new classification. Eur J Oral Implantol. 2011;4(3):227-43. 24- Mombelli A, van Oosten MA, Schurch E Jr, Land NP. The microbiota associated with successful or failing osseointegrated titanium implants. Oral Microbiol Immunol. 1987;2:145-51.

25- Renvert S, Aghazadeh A, Hallström H, Persson GR. Factors related to peri-implantitis - a retrospective study. Clin Oral Implants Res. 2014:25(4);522-9.

26- Rinke S, Ohl S, Ziebolz D, Lange K, Eickholz P. Prevalence of periimplant disease in partially edentulous patients: a practicebased cross-sectional study. Clin Oral Implants Res. 2011;22:82633.

27- Roos-Jansåker AM, Lindahl C, Renvert H, Renvert S. Nine to fourteen years follow-up of implant treatment. Part II: presence of peri-implant lesions. J Clin Periodontol. 2006;33:290-5.

28- Sanz M, Chapple IL, Working Group 4 of Seventh European Workshop on Periodontology. Clinical research on peri-implant diseases: consensus report of Working Group 4. J Clin Periodontol. 2012;39:202-6.

29- Schuldt Filho G, Dalago HR, Souza JG, Stanley K, Jovanovic $S$, Bianchini MA. Prevalence of peri-implantitis in patients with implant-supported fixed prostheses. Quintessence Int. 2014 Aug 12. doi:10.3290/i.qi.a32566. Epub ahead of print.

30- Serino G, Ström C. Peri-implantitis in partially edentulous patients: association with inadequate plaque control. Clin Oral Implants Res. 2009;20:169-74.

31- Souza JG, Neto AR, Filho GS, Dalago HR, Souza Júnior JM, Bianchini MA. Impact of local and systemic factors on additional peri-implant bone loss. Quintessence Int. 2013;44:415-24.

32- Tarnow DP, Cho SC, Wallace SS. The effect of inter-implant distance on the height of inter-implant bone crest. J Periodontol. 2000;71:546-9.

33- Van Winkelhoff AJ, Goené RJ, Benschop C, Folmer T. Early colonization of dental implants by putative periodontal pathogens in partially edentulous patients. Clin Oral Implants Res. 2000;11:511-20.

34- Wennström J, Palmer R. Consensus report of Section C. In: Lang NP, Karring T, Lindhe J, eds. Proceedings of the $3^{\text {rd }}$ European Workshop on Periodontology: Implant Dentistry. Berlin: Quintessenz; 1999. p. 255-9. 